\title{
Nutritional management of COVID-19 patients in a rehabilitation unit
}

\author{
Luigia Brugliera ${ }^{1}$ - Alfio Spina ${ }^{2} \cdot$ Paola Castellazzi $^{1} \cdot$ Paolo Cimino $^{1} \cdot$ Pietro Arcuri $^{3} \cdot$ Alessandra Negro $^{1}$. \\ Elise Houdayer ${ }^{1} \cdot$ Federica Alemanno $^{1} \cdot$ Alessandra Giordani $^{1} \cdot$ Pietro Mortini $^{2} \cdot$ Sandro Iannaccone $^{1}$
}

Received: 23 April 2020 / Revised: 2 May 2020 / Accepted: 6 May 2020 / Published online: 20 May 2020

(c) Springer Nature Limited 2020

Severe acute respiratory syndrome coronavirus 2 (SARS$\mathrm{CoV}-2$ ) has confounded the healthcare system organizations, especially in Italy, in the management of a dramatically increasing number of patients over a short period of time [1, 2]. Coronavirus disease 2019 (COVID-19) may cause interstitial pneumonia and respiratory distress syndrome, which can lead to multi-organ failure [3]. During the acute phase of COVID-19 pneumonia, patients may need noninvasive ventilation, CPAP or NIV, or endotracheal intubation [4]. Furthermore, COVID-19 seems to affect multiple organs, such as heart and kidney, and also to cause vascular damages facilitating thrombosis [5].

After the acute phase, specific rehabilitation should be performed to achieve respiratory improvement, functional and cognitive recovery, to decrease disability, and to improve quality of life [1].

Since the opening of our COVID-19 Rehabilitation Unit at the San Raffaele Scientific Institute (Milan, Italy) at the end of March 2020 (about 1 month ago), 50 patients have been admitted. Criteria for admission were positive nasopharyngeal swab test and functional independence measure (FIM) values showing dependence for motor or respiratory functions. The average FIM value in our cohort was 76.6. During the rehabilitation period, the swallowing function and the nutritional status of patients have been taken care of by a multidisciplinary team [1]. More than $90 \%$ of COVID19 patients in our cohort showed some degree of dysphagia,

Luigia Brugliera

brugliera.luigia@hsr.it

1 Department of Rehabilitation and Functional Recovery, I.R.C.C.S. San Raffaele Scientific Institute, Vita-Salute University, Milan, Italy

2 Department of Neurosurgery and Gamma Knife Radiosurgery, I.R. C.C.S. San Raffaele Scientific Institute, Vita-Salute University, Milan, Italy

3 Postgraduate Specialization in Physical and Rehabilitation Medicine, University of Milan, Milan, Italy with the need of a modified diet's consistency or nasogastric feeding. Previous communications reported a rate of dysphagia after orotracheal intubation of about $50 \%$. Our population reflects this rate [6]. These findings suggest that nutritional support and swallowing training are pivotal for the functional recovery of this particular type of patients. Moreover, adequate nutritional status allows for better resilience in the presence of pathological conditions, such as in the case of COVID-19 infection. Based upon the Malnutrition Universal Screen Tool (MUST), we found a high risk of malnutrition in $45 \%$ of our population (50 cases), whereas $26 \%$ of patients showed a moderate risk.

Recently, it has been reported that low prealbumin levels are strongly related to the rate of acute respiratory distress syndrome; therefore a worse nutritional status represents a negative prognostic factor for SARS-CoV-2 [3].

Respiratory syndrome of SARS-CoV-2 is often accompanied by prolonged immobilization, which can cause reduction of muscle functions until sarcopenia. Sarcopenia is related to an increased risk of malnutrition, disability, and more generally a worsening of the quality of life [1].

The risk of malnutrition of COVID-19 patients is related to chronic pathologies and the reduction of food intake caused by nausea, diarrhea, and the loss of appetite. The most common chronic conditions observed in COVID-19 patients are diabetes, chronic obstructive pulmonary disease, renal insufficiency, cardiovascular diseases, and dementia. Moreover, it is also well-known that advanced age is deeply related to the risk of malnutrition: according to the last published data by Italian Health Council regarding infections in Italy, about $40 \%$ of those infected by COVID19 are over 70 years old or more.

Some recent clinical communications have been published on the nutritional aspects of COVID-19 patients. Cintoni et al. supported a personalized meal supply protocol combined with oral food supplements to adequate the high energetic and proteic requirements induced by COVID-19, in those patients that can be fed orally. For those unable to eat, high proteins and enteral/parenteral nutrition (PN) 
formulas with low glucose content, according to recent ESPEN guidelines on clinical nutrition in the intensive care unit, are warranted [7].

Caccialanza et al. reported an early nutritional supplement protocol for noncritical COVID-19 patients. Their proposal is based on the observation that almost all patients present at the time of hospitalization had a severe inflammatory status and anorexia, which led to a strong reduction in food intake. Authors provided a rapid intravenous administration of whey proteins, vitamins, and minerals (and cholecalciferol if insufficient) up to the achievement of the recommended dietary allowance [8]. Furthermore, the authors assessed the nutritional status early with consequent addition of oral food supplements or, if not tolerated, transition to artificial nutrition [8].

It has already been demonstrated that malnutrition delays healing times and increases hospitalization periods. Therefore, the prevention, diagnosis, and treatment of malnutrition must be regularly included in the management of hospitalized COVID-19 patients in a rehabilitation department, to improve both short and long-term prognosis $[9,10]$. Malnutrition must be considered as the inability to preserve a correct body composition and muscle mass, and not necessarily related to a low body mass index. For this reason, also obese patients have to be considered according to the same criteria. Moreover, it is noteworthy that a personalized swallowing rehabilitation targeting the recovery of normal feeding should be provided during the hospitalization, in order to reduce the duration of admissions [11].

Collaboration between the Rehabilitation Unit of the San Raffaele Scientific Institute and the dietetics service created an interdisciplinary and integrated management of the nutritional status of COVID-19 patients, based on the latest scientific data. We report here this three-step nutritional protocol.

\section{Step 1-nutritional assessment and malnutrition screening}

All hospitalized COVID-19 patients must undergo a meticulous nutritional assessment and a malnutrition screening.

The nutritional evaluation must consider the following aspects:

(1) Anthropometric parameters. Using a scale, if the patient is able to maintain the upright position, or alternatively by using a scale chair, the body weight is evaluated. Weight can be estimated only if a direct measurement is not possible because of immobilization. Height can be reported by the patient or directly measured.

(2) Impedance and vector analysis. This examination is useful to analyse patients' body composition, by comparing data with standard references. It is possible to quantify muscle mass, external and internal total cell hydration, and other nutritionally relevant parameters such as the phase angle [12].

(3) Weight loss. Direct patient interview or according to clinical report.

(4) Hematochemical parameters. Blood count, PCR, total proteins, serum protein electrophoresis, prealbumin, ferritin, sideremia, folic acid, B12 vitamin, blood sugar, electrolytes, and liver function indices can be used in the analysis of the nutritional status.

(5) Swallowing. Medical record and ENT evaluation allow to identify a specific diet according to the swallowing ability. Patients unable to eat must undergo artificial feeding, according to the AN management guidelines.

(6) Intake assessment. A food diary may be useful to register food intake during the hospitalization.

A reference-screening tool for patients is represented by the NRS-2002 test, which is performed on all hospitalized patients. Patients undergo the MUST at hospital admission and discharge.

\section{Step 2-setting the nutritional treatment}

\section{Requirements}

(1) Energy requirement. Because of the impossibility of using indirect calorimeter, the daily requirement must be estimated through predictive equations based on body weight, such as $27-30 \mathrm{kcal} / \mathrm{kg} \backslash$ day, adapted on the personal nutritional status, level of physical activities, clinical status, and comorbidities.

(2) Protein requirement. In case of the absence of chronic renal insufficiency, the protein intake is $>1 \mathrm{~g}$ kglday (up to $1.5 \mathrm{~g} \mid \mathrm{kg} \backslash$ day). This must be adapted to the aforementioned features as for energetic intake, in order to prevent weight loss, reduce the risk of complications, and promote a global recovery.

(3) Carbohydrate and lipid requirements. It must refer to the nonprotein energy requirement, with a lipids/ carbohydrates ratio ranging from 30:70 (patients without respiratory insufficiency) to 50:50 (patients with respiratory insufficiency). Low glycaemic index carbohydrates have to be considered.

(4) Water requirement. Adequate hydration must be maintained according to remote (heart or renal failure) and recent clinical history (diarrhea, vomiting, electrolyte imbalances). 


\section{Diet setting}

(1) Adequate diet for each patient is guaranteed by the hospital computerized meal management system.

(2) Patients' dietary assignment is shared with all the involved healthcare professionals.

(3) Nutritional advices that can be followed by the patient in the hospital and after home discharge is warranted.

\section{Additional supportive therapy}

(1) Adequate vitamins and oligoelements integration must be warranted [13].

(2) Essential and branched aminoacids should always be considered for rehabilitation patients [13].

(3) Probiotics must be considered, particularly in case of intestinal issues.

(4) During hospitalization, oral nutritional supplements (ONS) are useful in case of malnutrition or for those cases which intake is only $50-60 \%$ of the programmed one. In case of dysphagia, it is mandatory to modify diet consistency in addition to ONS supplementation.

\section{Artificial nutrition}

If spontaneous supplies are less than 50-60\% and spontaneous oral nutrition is expected to be impossible for more than 3 days, artificial nutrition must be started. Enteral nutrition (EN) has to be preferred to maintain muscle tone, microbiota, and intestinal immunity as well as for the lower risk of infectious complications compared to PN [10]. The choice of the appropriate nutritional mixture must be individualized. In all cases, potential complications of EN should be monitored. Gastrostomy is the standard approach for EN administration, starting with $20 \mathrm{ml} / \mathrm{h}$ to gradual increase until the nutritional goal is reached [14]. Adverse reaction of $\mathrm{EN}$ has to be considered in the light of the gastrointestinal manifestations of SARS-CoV-2. Those patients who underwent EN, have reported higher rates of respiratory complications and longer durations of NIV application, compared with those with no EN [8]. In these patients, $\mathrm{PN}$ is preferred to $\mathrm{EN}$ since nasogastric can cause air leaks and compromise the effectiveness of NIV or CPAP [15].

\section{Step 3-continuous monitoring}

Body weight, intake, blood tests, and clinical condition are monitored by the multidisciplinary team over time with variable frequency. Custom protocol changes are performed based on clinical evolution. Outpatient continuous evaluations are provided.

Concerning the preliminary data among 32 patients with at least 10 days of hospitalization in the COVID-19 rehabilitation unit, MUST improved in five patients (15.3\%), while in the remaining it did not change. No deaths were reported.

Fourteen patients $(43.7 \%)$ experienced BMI improvement, while in 15 cases $(46.8 \%)$ BMI was stable. The mean BMI value was $20.31(\mathrm{SD} \pm 5.84)$ at unit admission, while 23.94 $(\mathrm{SD} \pm 5.79)$ at discharge; the difference was found statistically significant [paired $t$-test; $t[31]=2.498 ; p=0.018$ ].

The implementation of a systematic management of the nutritional intake of COVID-19 patients is essential to guarantee an optimal nutritional status and to improve clinical outcomes. Further clinical studies are on-going to draw up adequate nutritional protocols. Nutrition is a determining factor for health. Underestimating the importance of nutrition in COVID-19 patients is an error that can dramatically affect the outcome of these patients who are suffering from a devastating and disabling disease. Nutritional support is paramount together with the rehabilitation to improve the chances of recovery in COVID-19 patients.

\section{Compliance with ethical standards}

Conflict of interest The authors declare that they have no conflict of interest.

Publisher's note Springer Nature remains neutral with regard to jurisdictional claims in published maps and institutional affiliations.

\section{References}

1. Brugliera L, Spina A, Castellazzi P, Cimino P, Tettamanti A, Houdayer E, et al. Rehabilitation of COVID-19 patients. J rehabilitation Med 2020;52:jrm00046.

2. Zangrillo A, Beretta L, Silvani P, Colombo S, Scandroglio AM, Dell'Acqua A, et al. Fast reshaping of intensive care unit facilities in a large metropolitan hospital in Milan, Italy: facing the COVID-19 pandemic emergency. Crit Care Resusc. 2020 [Epub ahead of print].

3. Zhou F, Yu T, Du R, Fan G, Liu Y, Liu Z, et al. Clinical course and risk factors for mortality of adult inpatients with COVID-19 in Wuhan, China: a retrospective cohort study. Lancet. 2020;395: 1054-62.

4. Ding L, Wang L, Ma W, He H. Efficacy and safety of early prone positioning combined with HFNC or NIV in moderate to severe ARDS: a multi-center prospective cohort study. Crit Care. 2020;24:28. 
5. Guan WJ, Ni ZY, Hu Y, Liang WH, Ou CQ, He JX, et al. Clinical characteristics of coronavirus disease 2019 in China. N Engl J Med. 2020;382:1708-20.

6. Brodsky MB, Levy MJ, Jedlanek E, Pandian V, Blackford B, Price $\mathrm{C}$, et al. Laryngeal injury and upper airway symptoms after oral endotracheal intubation with mechanical ventilation during critical care: a systematic review. Crit Care Med. 2018;46:2010-7.

7. Cintoni M, Rinninella E, Annetta MG, Mele MC. Nutritional management in hospital setting during SARS-CoV-2 pandemic: a real-life experience. Eur J Clin Nutr. 2020;74:846-7.

8. Caccialanza R, Laviano A, Lobascio F, Montagna E, Bruno R, Ludovisi $\mathrm{S}$, et al. Early nutritional supplementation in noncritically ill patients hospitalized for the 2019 novel coronavirus disease (COVID-19): rationale and feasibility of a shared prag-matic protocol. Nutrition. 2020:110835 [Epub ahead of print].

9. Barazzoni R, Bischoff SC, Breda J, Wickramasinghe K, Krznaric $Z$, Nitzan D, et al. ESPEN expert statements and practical guidance for nutritional management of individuals with SARS-CoV-
2 infection. Clin Nutr. 2020; pii: S0261-5614(20)30140-0 [Epub ahead of print].

10. Volkert D, Beck AM, Cederholm T, Cruz-Jentoft A, Goisser S, Hooper L, et al. ESPEN guideline on clinical nutrition and hydration in geriatrics. Clin Nutr. 2019;38:10-47.

11. Covinsky K, Katz M. Supplemental nutrition assistance program do not take the food out of patients' mouths. JAMA Intern Med. 2020 [Epub ahead of print].

12. Kyle UG, Bosaeus I, De Lorenzo AD, Deurenberg P, Elia M, Gomez JM, et al. Bioelectrical impedance analysis-part I: review of principles and methods. Clin Nutr. 2004;23:1226-43.

13. Zhang L, Liu Y. Potential interventions for novel coronavirus in China: a systematic review. J Med Virol. 2020;92:479-90.

14. Bankhead R, Boullata J, Brantley S, Corkins M, Guenter P, Krenitsky J, et al. Enteral nutrition practice recommendations. J Parenter Enter Nutr. 2009;33:122-67.

15. Singer $P$, Rattanachaiwong $S$. To eat or to breathe? The answer is both! Nutritional management during noninvasive ventilation. Crit Care. 2018;22:27. 